\title{
Hybrid control of supply chains: a structured exploration from a systems perspective
}

\author{
Grefen, P., \& Dijkman R. \\ School of Industrial Engineering, Eindhoven University of Technology \\ P.W.P.J.Grefen@tue.nl \\ R.M.Dijkman@tue.nl
}

\begin{abstract}
Supply chains are becoming increasingly complex these days, both in the structure of the chains and in the need for fine-grained, real-time control. This development occurs in many industries, such as manufacturing, logistics, and the service industry. The increasing structural complexity is caused by larger numbers of participating companies in supply chains because of increasing complexity of products and services. Increasing requirements to control are caused by developments like mass-customization, pressure on delivery times, and smaller margins for waste. Maintaining well-structured strategic, tactic, and operational control over these complex supply chains is not an easy task - certainly as they are pressured by end-to-end synchronization requirements and just-in-time demands. Things become even more complex when chains need to be flexible to react to changing requirements to the products or services they deliver. To enable design of well-structured control, clear models of control topologies are required. In this paper, we address this need by exploring supply chain control topologies in an organized fashion. The exploration is based on integrating a supply chain model and a control model in two alternative ways to obtain two extreme models for supply chain control. These two models are next combined to obtain a hybrid chain control model in which control parameters can be adapted to accommodate different circumstances, hence facilitating agility in supply chains and networks. We apply the developed model to a number of case studies to show its usability. The contribution of this paper is the structured analysis of the design space for chain-level control models - not the description of individual new models.
\end{abstract}

Key words: supply chain, control model, information system.

\section{Introduction}

In the modern economy, we see the development of ever more complex products. A good example can be found in the automotive domain. Here, we see that the complexity of automobiles has increased in a dramatic way: the inclusion of new features like safety systems, driver guidance and support systems, mobile entertainment systems, and air conditioning systems has increased the number of components in an average car significantly (Maxton and Wormald, 2004). Similar developments can be observed in many high-tech industries, like the airplane industry and the computer industry.

Next to the increasing complexity of products, we also see an increasing variety of products: many products cannot be delivered anymore as a standard product that uniformly suits every customer. These products are customized for specific markets, for specific customer groups or even individual customers giving rise to the development of mass customization in the past decades (Smith, Jiao and Chu, 2012). We can find a good example in the automotive industry again where each individual car is delivered to the requirements of an individual customer.

Apart from the increasing complexity and variety of products, we also see that the pressure on performance of supply chains increases. Delivery times need to be compressed to stay competitive in modern markets that behave increasingly in a real-time fashion. Increasing competition and the advent of 'green' chains increase the pressure on waste reduction, for example to decrease the carbon footprint of a chain (Hoen et al., 2012). This requires tight integration of a complex chain, e.g., in multimodal logistics (Jansen et al., 2004). 
On top of the complexity introduced by the above static aspects of supply chains, chains must also be able to react quickly to changes in their environments. A typical cause on the strategic or tactic decision level is the shortening life cycle of many products and services. Changing requirements to products or services can imply reorganizations of supply chains (e.g., because a different composition of modules in a product is required). A typical cause on the operational level is found in exceptions occurring during supply chain execution (such as transport disruptions), giving rise to synchro-modal logistics in which alternative transportation modes can be used side-by-side.

The above developments in markets have a profound influence on the way companies collaborate to serve their customers:

1. The complexity of products requires that the organizations that produce them need to collaborate in complex production supply chains, where each partner in a chain contributes to part of the complex product (Corswant and Frederiksson, 2002). Chains can have a simple linear structure, but can also have complex network structures. In a survey by the Aberdeen Group, growing supply chain complexity is identified as the top business pressure (Heaney, 2012).

2. The variety of products requires that a supply chain reacts as early as possible to the requirements of the customer, pushing customerspecific operations as far as possible towards the start of the chain. This transforms a supply chain into a demand chain (or a hybrid form in between).

3. Pressure on delivery times and conditions requires that a supply chain must operate in a (near) real-time fashion such that delays can be avoided and exceptions can be handled on-thefly.

4. The short life cycle of products requires that supply chains must be able to change in a fast and efficient way to produce new (generations of) products to be able to serve markets. This may lead to highly dynamic chains and networks or even instant virtual enterprises (Grefen et al., 2009; Mehandjiev and Grefen, 2010).

These developments in business collaboration require a high level of control to enable effective and efficient operation of complex supply chains. In the past, operation control was centered on intra-organizational issues and inter-organizational issues were considered as simple, messagebased synchronization points between the 'intraorganizational islands' of control. In the modern situation, the emphasis needs to be on interorganizational control as well - in some situations perhaps even more than on the intra-organizational control: the operation of the chain as a whole often determines business success more than the operation of the individual links of the chain.

This paper addresses the issue of chain control in the context explained above. The aim of the paper is to provide a simple conceptual framework that can serve as the basis for analysis and design of complex chain control. As information is the basis for control, the paper takes an information system perspective in its analysis. We take a qualitative approach in this paper, but this can be extended into a quantitative approach.

The structure of this paper is as follows. First, we lay the basis for the framework in a simple supply chain model (Section 2) and a simple business control model (Section 3). Next, we integrate these two models in two ways to arrive at two extreme chain control models. We first discuss a decentralized control model in Section 4, which is in many cases the current default in practice. We show what the weaknesses of this model are in terms of transparency and synchronization. Secondly, we present a model with centralized control in Section 5. This model avoids the weaknesses of the model with decentralized control. Centralized control, however, has its own weaknesses in terms of autonomy and vulnerability (which are in turn avoided by the decentralized approach). To address the weaknesses of both extreme models, we combine the decentralized and centralized approaches into a model with hybrid chain control in Section 6 . This model can be used in a parameterized way to be adapted to a specific context. In Section 7, we focus on agility in this hybrid model, i.e., on ways to organize an implementation of this model such, that change becomes a structural, natural element of this implementation, instead of a disruptive factor. In Section 8, we present case studies in supply chain developments to illustrate the developed idea from a more practical perspective and show its applicability. We end this paper with conclusions in Section 9.

\section{Basic supply chain model}

In this section, we present a basic supply chain model that we use as the first ingredient for the supply chain control model that we develop in the sequel of this 
paper. We base the discussion on linear chains for reasons of simplicity, but the approach applies to arbitrary business network topologies, as discussed for example by Grefen et al. (2009), and Mehandjiev and Grefen (2010).

\subsection{The basic model}

In Figure 1, we show the basic supply chain model that we use in this paper (this model can be considered an abstracted variation on the model used in Muckstadt et al. (2001)). We use a supply chain consisting of three links for reasons of presentation clarity, where each link represents an autonomous organization in the supply chain. Three links include a start link, a middle link, and an end link. Longer chains can be formed by having multiple middle links, but this does not change the approach of this paper.

In the figure, the double arrows indicate the material flows. The supply chain receives material input from its environment (e.g., raw materials) and delivers its output to this environment (e.g., end products). In manufacturing and logistics industries, materials are typically of a physical nature (like car parts in the automotive industry). In the service industry, like the financial industry, materials may also be non-physical (like elements of complex financial products).

The single arrows indicate information flows related to the execution of business processes. Order (purchase) process info flows from end to start of the supply chain to initiate the delivery of materials. Delivery process info flows from start to end to organize the delivery of materials. Delivery process info covers both information on the nature of the products delivered (such as product identification and quantity) and information about their transport (such as delivery times). Detailed material documentation is typically transferred as part of the material flow.

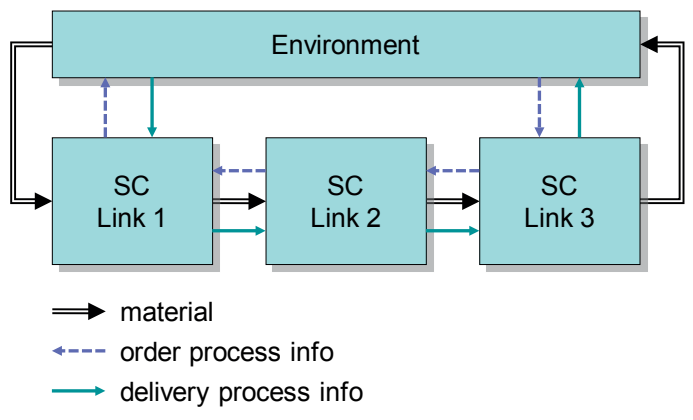

Figure 1. Basic supply chain model with material and information flow structure.

\subsection{Supply chains $v$ s. demand chains}

In pure supply chains, large batch-wise purchase orders are typically issued periodically by supply chain links to replenish stocks at these links, such that operation of the individual links is decoupled as much as possible.

In pure demand chains, all purchase orders in the chain are triggered by individual purchase orders originating from the environment. Demand chain operation couples the operation of individual links in the chain in a fine-grained fashion, thereby introducing high demands on synchronization of activities between supply chain links. Insufficient inter-link synchronization introduces inter-link problems with respect to the effectiveness and efficiency of the chain.

In complex chains, we see hybrid situations where the upstream part of the chain operates in supply chain mode (make to stock) and the downstream part in demand chain mode (make to order). The point where upstream and downstream parts meet is called the customer order decoupling point (CODP) (Olhager, 2012). In modern markets, chains are increasingly changing from supply chain to demand chain operation by moving the COPD more upstream.

\subsection{Internal structure of chain links}

Each of the links in the supply chain model of Figure 1 can be modeled by Porter's value chain model (Porter, 1985). We see the result of this modeling in Figure 2 with Porter's model slightly simplified as in Grefen (2010). The material and process information flows in the figure coincide with those in Figure 1.

From Figure 2, we can see that within a single link, four different business functions are involved

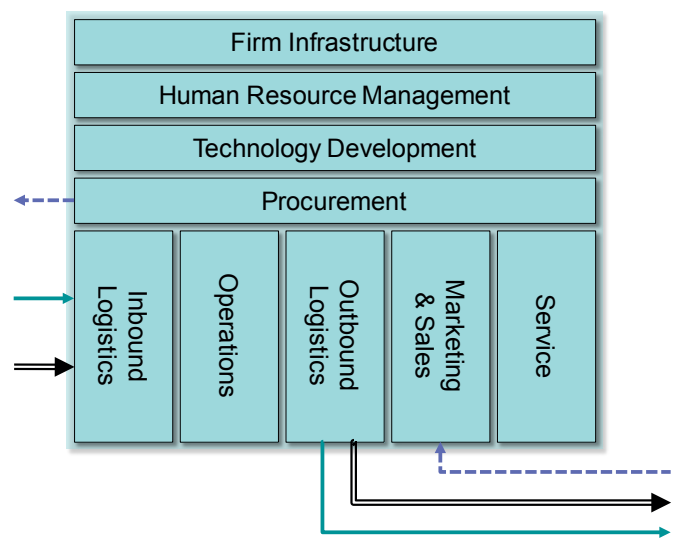

Figure 2. Supply chain link with internal structure. 
in supply chain control. This may create intra-link synchronization problems, where synchronization is not fine-grained enough. These intra-link problems come on top of the inter-link problems we have observed before in this section.

Note that Porter's model was originally developed for the manufacturing industry. It can be applied, however, with slight modification, to other industry domains. In case of a logistics company, for example, the operations function refers to transport and the inbound and outbound logistics to respectively loading and unloading. We will see different industry domains when we get to our application case studies in Section 8.

\section{Basic control model}

After introducing our basic supply chain model in the previous section, we now introduce our second main ingredient: the basic control model. We first present the model itself. Then, we focus on the two types of control loops in the model. Next, we introduce control levels in terms of time horizons.

\subsection{The basic model}

The basic control model we use is shown in Figure 3. This model is a simple cybernetic model with an emphasis on information processing. Double arrows are material (product) flows, single arrows information flows (as in the basic supply chain model introduced in the previous section).

The model consists of an environment, a transformation system, a control system, and an information system. The latter three components form the business organization under study. The transformation system receives materials from the

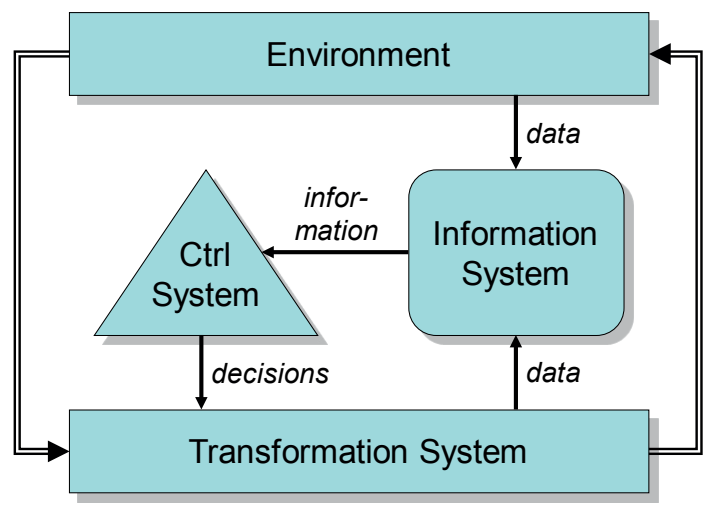

Figure 3. Basic control model. environment and transforms them into other materials which it feeds again to the environment. Typically, the transformation adds value to the materials. The precise nature of the transformation depends on the industrial context: it can be manufacturing, transport or service integration, for example. The control system controls the transformation system, i.e., it takes decisions about its operation. To take these decisions, it receives information from the information system. The information system produces information based on data it retrieves from both the transformation system (internal data) and the environment (external data).

\subsection{Control loops}

In our basic control model, control loops are used to regulate the behavior of the transformation system. A control loop includes making a decision, executing it in the transformation system, observing its effects, and deciding about its effectiveness. We can distinguish between two control loops in the basic

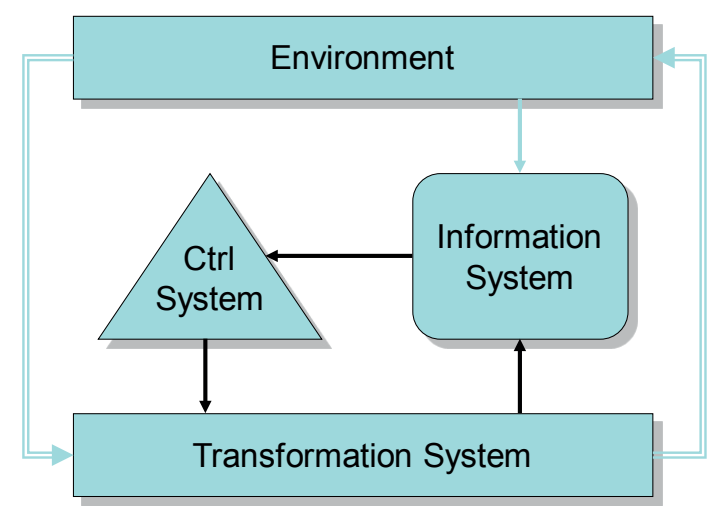

Figure 4. Intern control loop.

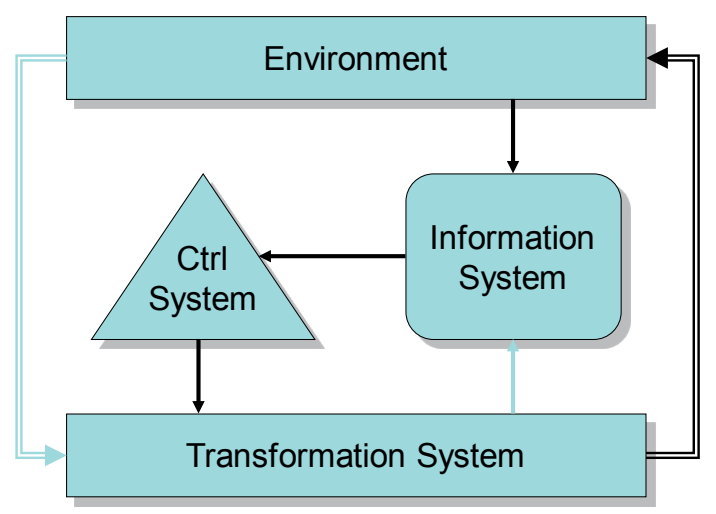

Figure 5. External control loop. 
control model: the internal loop and the external loop (as illustrated in Figure 4 respectively Figure 5).

The internal loop is completed within the boundaries of the organization under study (it is an intraorganizational loop). The control loop is used to adjust the transformation system to meet internal goals. The cycle time of the loop is fully under the control of the organization. The minimum cycle time for the internal loop is equal to the sum of the time taken to process information in the information system, the time taken to make a decision in the control system, and the time to implement this decision in the transformation system.

The external loop includes the environment: the information system retrieves data about the response of the environment to materials produced by the transformation system. The control loop is used to adjust the transformation system to meet external goals. The minimum cycle time of the external loop is dependent on the reaction time of the environment and is hence not fully under the control of the organization. The cycle time is also dependent on the time to feed the output of the transformation system to the environment and on the time to retrieve information from the environment into the information system.

Obviously, internal and external control loops can be combined to serve different purposes in the context of business management.

\subsection{Control levels}

The control system introduced in Section 3.1 operates at three control levels (as illustrated in Figure 6), each of which has its own time horizon.

The lowest level is the operational level. At this level, processing of individual client orders is controlled.

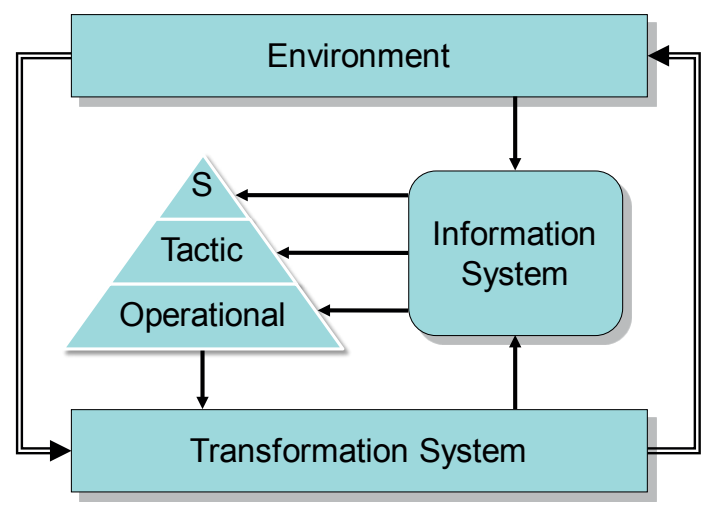

Figure 6. Control levels (time horizon).
The time horizon is short. Many individual decisions have to be taken, but these are typically of a routine character. Only in collaborations that change on a per-client-order basis (dynamic collaborations according to Grefen (2010)), decisions that affect the mode of collaboration are taken at this level.

The middle level is the tactical level. This level is concerned with handling of batches of client orders. The time horizon is medium. The amount of decisions is also medium, but these decisions require more intelligence. Often, these decisions imply setting the parameters for the operational level. Also, the selection of tactical supply chain partners (in semi-dynamic collaborations according to Grefen (2010)) belongs to this level.

The highest level is the strategic level. Here, decisions are taken that are not related to concrete orders - implementing the business strategy is at stake here. Selecting strategic partners (in static collaborations according to Grefen (2010)) is a main issue at the strategic level. Here, the parameters for the tactical level are set.

Each level requires information at the appropriate level (as indicated by the three arrows in Figure 6). Information for the strategic level is most aggregated and abstract, that for the operational level most detailed and concrete. As such, the model shares characteristics with the Viable System Model proposed by Beer (1984), in which also a hierarchy of control systems is used (applied in supply chain management for example by Verdouw at al. (2011)).

\section{Chains with decentralized control}

In this section, we discuss the first and most common case of supply chain control: the case of fully decentralized control. This case is most common because it is based on autonomous links in the chain that collaborate on the basis of link-to-link (peer-topeer) message passing.

We start this section with constructing our supply chain control model by combining the two basic models that we have introduced in the two preceding sections.

\subsection{The supply chain control model with decentralized control}

To obtain a supply chain control model with decentralized control, we combine the basic supply chain model of Figure 1 and the basic control model of Figure 3. 
To do so, we first have to answer the question how the two basic models are combined. As all links in a chain with decentralized control are fully autonomous, they each have their own control system. This implies that the basic control model will be embedded in the basic chain model on a per-link basis. Consequently, we construct the model by replacing each black box link of the basic supply chain model by the structure of the basic control model. In modeling terms, we substitute and refine each of the links in the supply chain.

Next, we have to answer the question where to place the inter-link flows of the basic chain model between the elements of the control model instances. To answer this question, we map Porter's model (see Figure 2) to the basic control model. The transformation system module of the control system should be mapped to all business functions related to supply chain management and production, i.e., all primary functions plus procurement. The control system module of the control model is in control of the entire transformation system. Hence, it must be placed 'above' these functions. The information system module is at the same level as the control system module. This implies that we map these two modules to the firm infrastructure function. These choices are illustrated in Figure 7.

Afterhaving answered the above two questions, we can construct the supply chain control model. The resulting model is shown in Figure 8. For reasons of simplicity and clarity, we have not distinguished between the two kinds of information flow (purchase orders and product information, see Figure 1) in this figure.

Note that in the model of Figure 8, all inter-link information flow occurs between transformation systems of the individual links (conforming to Figure 2 and Figure 7). Likewise, there are

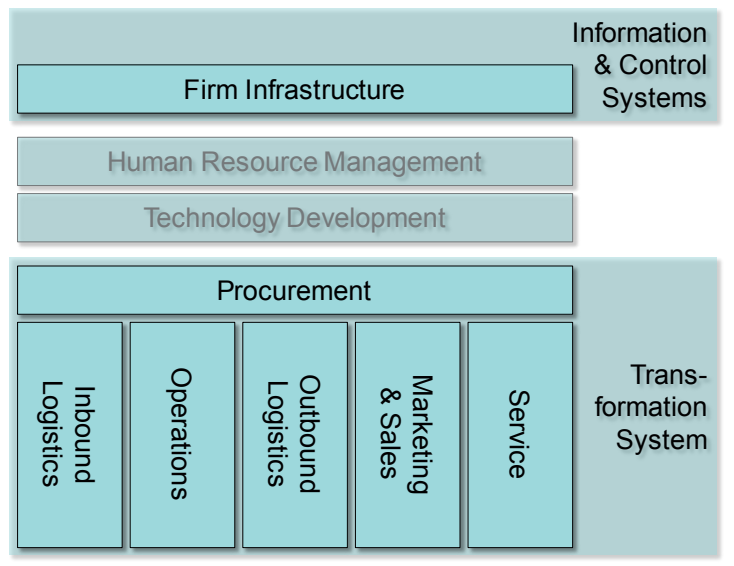

Figure 7. Mapping of Porter's functions to basic control model.

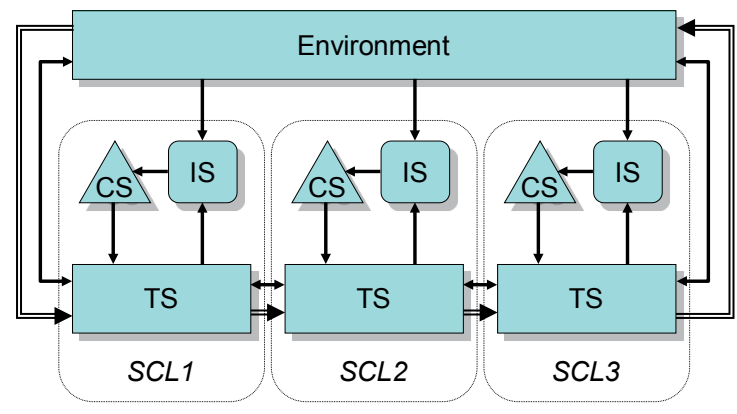

Figure 8. Basic supply chain control model with control information passing at TS level.

information flows between the environment and the transformation systems of the first and last links of the chain (to transport the order process information and delivery process information of Figure 1). The information flows between the environment and the information systems of the chain links transport additional contextual business information.

\subsection{Lifting the level of control information passing}

The model of Figure 8 implies that all inter-link information has to pass through various business functions of the individual links - as observed in the discussion of Figure 2. As this is undesirable for reasons of efficiency, we can 'lift' the level of inter-link information passing to the level of the control and information systems of the chain links. The resulting model is shown in Figure 9. Note that we have included information flows from the control systems of the first and the last chain links to the environment. These information flows correspond with the information flows from the transformation systems of the first and last chain links to the environment in Figure 8 (the upward direction of the two flows in the figure). The reverse information flows are merged with the flows from environment to information systems in Figure 9.

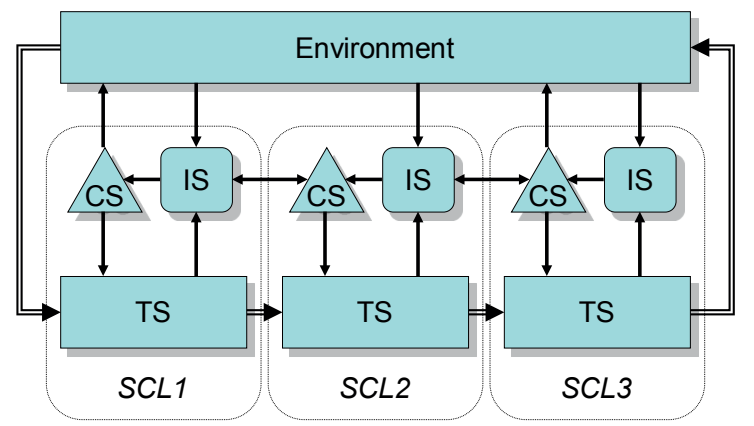

Figure 9. Basic supply chain control model with explicit control information passing. 
The model of Figure 9 explicitly distinguishes between the level of the primary process (the transformation systems) and the level of the control process (the control systems and information systems, including the intra- and inter-organizational connections between them), comparable to other supply chain control models (e.g., Verdouw et al. (2011)).

In terms of distributed business service management, the topologies shown in Figure 8 and Figure 9 are cases of choreography (Aalst 2009a): we have peerto-peer control to make a global business process work.

\subsection{Chain-level control loops}

In Section 3.2, we have discussed the internal and external control loops in the basic control model. As the supply chain control model is constructed using this basic control model, we can identify corresponding control loops in this model, but now on the chain level.

An internal chain-level control loop is used to manage the internal operation of the supply chain, i.e., without involving the environment. We can have variations on chain-level control loops. A full chainlevel internal control loop is shown in Figure 10. In this loop, materials are fed forward from the start through the chain and information about requirements to materials (like ordering information) is fed backward from the end through the chain.

The minimum cycle time of the loop depends on the sum of the internal processing times (both forward in TS and backward in IS and CS) of the individual links plus the communication times between the links (both forward communication at the TS level and backward communication at the IS/CS level). Obviously, the longer a chain is, the longer the minimum cycle time is.

An external chain-level control loop is used to manage the external operation of the entire chain,

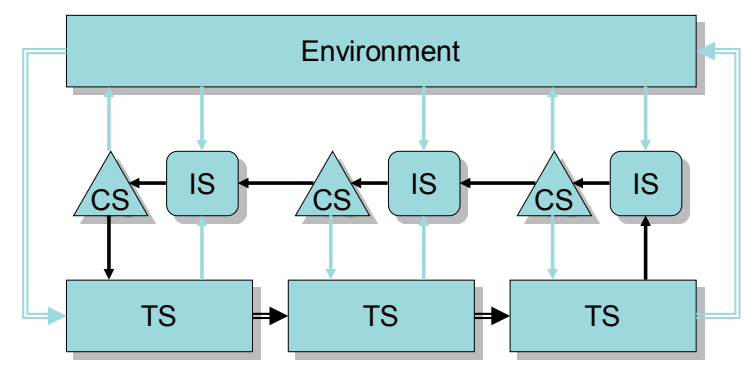

Figure 10. Full internal chain-level control loop in decentralized control model. i.e., to have the chain react to responses from the environment. Again, we can have several variations on control loops, two extremes of which we illustrate.

Figure 11 shows a configuration where feedback from the environment is fed back into the chain as soon as possible, i.e., at the tail of the chain. This configuration is used in a situation where the link that delivers the end products to the environment also monitors the reaction of the environment to these products (e.g., in a typical production chain). In this configuration, for the other links of the chain, the situation is as for the internal control loop.

Figure 12 shows an external control loop where the feedback from the environment is fed back into the head of the chain. This configuration is applicable in a situation where the producer of the initial materials monitors the reaction of the environment to these materials (e.g., in a typical logistics chain). In this configuration, the other links are not involved at all in the control loop at the CS and IS level.

\subsection{Evaluation of chains with decentralized control}

For chains with decentralized control, minimal control cycle times are heavily dependent on the length of the chain. This means that for chains with non-trivial lengths, several problems can arise:

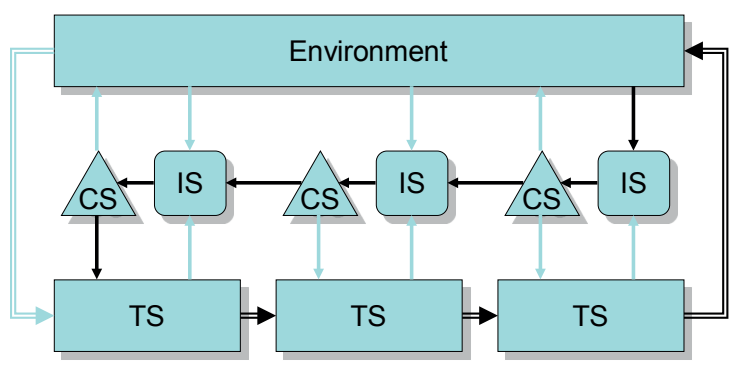

Figure 11. External chain-level control loop in decentralized control model (minimal external scope).

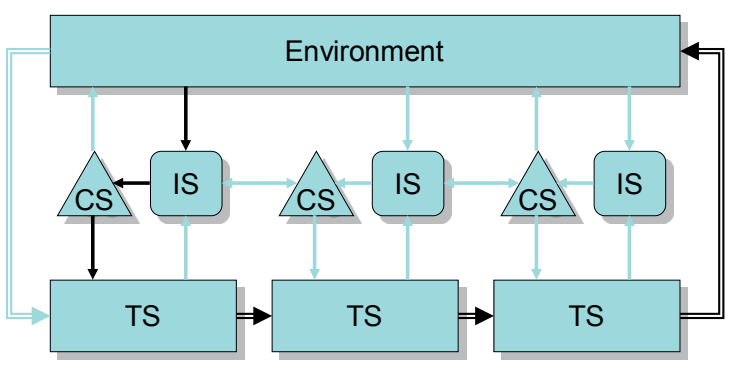

Figure 12. External chain-level control loop in decentralized control model (maximal external scope). 
- The chain becomes non-reactive to internal events.

- The chain becomes non-reactive to external events.

- The chain becomes confused by concurrent events, the handling of which may 'catch up' with each other.

- The chain may miss important information because links are 'skipped' in information processing (as in Figure 12).

Often, links in the chain have only limited visibility with respect to processes is the entire chain (Schulte et al., 2012), which may lead to sub-optimization.

Because of the above problems, the principle of decentralized control may be departed from. If we depart from it completely, we arrive at chains with fully centralized control. We discuss these in the next section.

\section{Chains with centralized control}

Given the drawbacks of decentralized chain control as discussed in the previous section, we now move to the opposite control paradigm: fully central chain control. We first discuss the chain control model with centralized control. Next, we discuss the internal and external control loops in this model. We end this section with an evaluation of the model (like in the previous section).

\subsection{Supply chain control model with centralized control}

In a chain with centralized control, there is one control system that controls the entire composite transformation system, i.e., the combination of the transformation systems of the entire chain. To construct a control model for this situation, we embed the supply chain model into the control model (opposite to what we have done to construct the decentralized control model in the previous section): we substitute the black-box transformation system of Figure 3 with the chain of Figure 1.

This means that in the supply chain control model with centralized control, we move the information system and control system functionality from the local level (per chain link) to the chain level. Consequently, we need only one chain-level control system (CCS) and one chain-level information system (CIS), as obtained by embedding the basic chain model into the basic control model. The result is shown in Figure 13 (for reasons of clarity, data/ information links are represented by dashed lines,

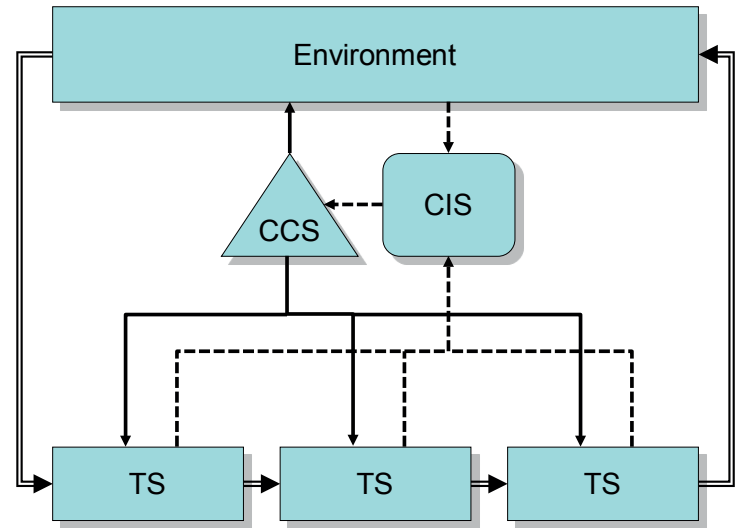

Figure 13. Chain control model with fully centralized control.

decision links by solid lines). Note that a decision flow is included between CCS and environment to implement the order process information flow of Figure 1.

The CIS provides the information in the chain. To do so, it performs two kinds of monitoring. In the first place, it monitors the internal operation of the entire chain. Hence, it can create aggregated information that individual links cannot create. This can be used to improve the effectiveness of the chain. In the second place, it monitors the environment on behalf of the entire chain. This avoids replication of this task in the individual links. This yields an improvement of efficiency of the chain.

The CCS controls the transformation systems of the individual links in the chain, thereby synchronizing their operation. This creates two possibilities. Firstly, the CCS can synchronize activities of individual transformation systems with information at the chain level (which individual links do not have available). Secondly, the CCS can manage business activities executed across individual links (which is hard to realize at the local level). In terms of distributed business service management, this is a case of business service orchestration (Aalst 2009b): one central component controls the execution of a number of participants in a business process.

\subsection{Control loops in the centralized chain control model}

In the centralized chain control model, we can identify chain-level internal and external control loops as introduced in Section 3.2.

The internal chain-level control loop is shown in Figure 14. 


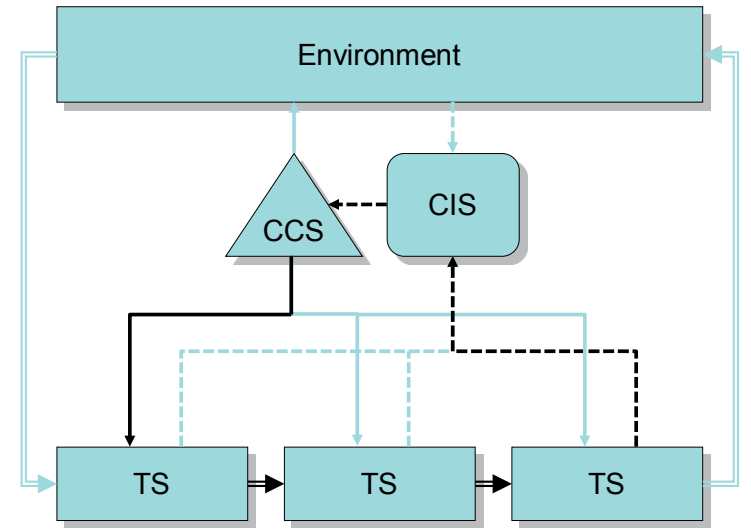

Figure 14. Internal chain-level control loop in centralized control model.

With external chain-level control loops, we can distinguish between minimal and maximal external scope, as we have done for the decentralized control model. The resulting loops in the centralized control model are shown in Figure 15 and Figure 16.

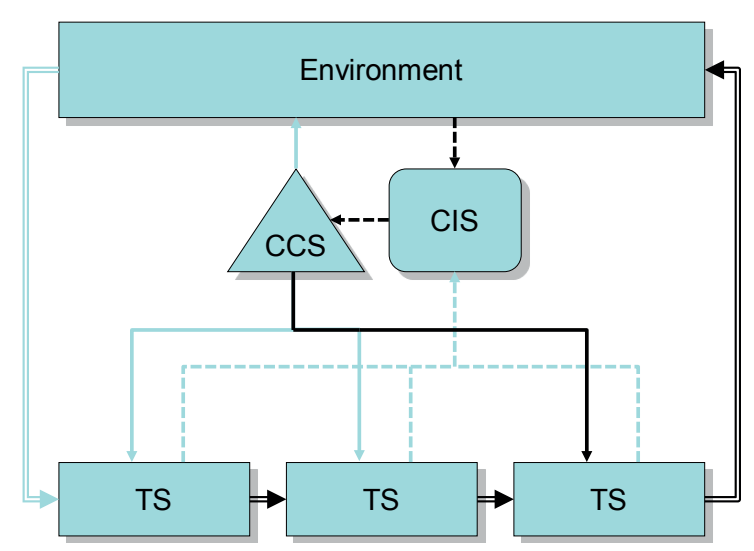

Figure 15. External chain-level control loop in centralized control model (minimal external scope).

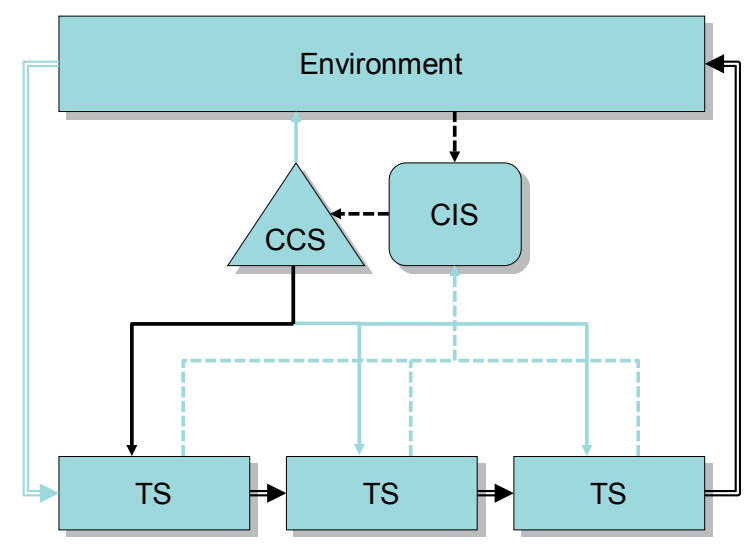

Figure 16. External chain-level control loop in centralized control model (maximal external scope).
In a concrete scenario, the CCS can decide where to send its decisions into the chain, i.e., to vary in the spectrum of minimal and maximal external scope of the control loop to optimize effectiveness or efficiency of the chain. This provides a higher level of information routing flexibility than in the decentralized control model of Figure 11 and Figure 12.

\subsection{Evaluation of the centralized chain control model}

The obvious advantage of the centralized model is that chain control becomes much easier and more transparent. We have also seen that information routing can be more flexible.

The obvious disadvantage is that link autonomy completely disappears. This has important consequences:

- More information is shared between organizations (and processed by the CIS), which may affect competitiveness of individual participants. The more dynamic chains are (the more their composition is changed depending in market circumstances), the more important this may be.

- There is a risk of 'one-size-fits-all' decision making, which may not adequately take into account specific characteristics of individual organizations in a chain.

- Each of the organizations in the chain has to completely trust the CCS in its decision making, certainly where individual organizations in the chain may have conflicting interests.

Depending on the organizational nature of the combination of CCS and CIS, the weight of the above issues varies. The CCS+CIS system may be an independent party or may be owned by one of the organizations in the chain (typically the most powerful one). If the CCS+CIS system is independent, it may either be a trusted third party (TTP, for example semi-government) or another commercial partner with interests of its own. The latter case becomes certainly interesting if the service-dominant business paradigm (Lusch, Fargo and O'Brien, 2007) is applied in the formation of service-dominant business networks (Lüftenegger, Grefen and Weisleder, 2012), where the orchestrator may actually be the most important player in the network.

A compromise to address the last two issues outlined above can be found in a model with a centralized 


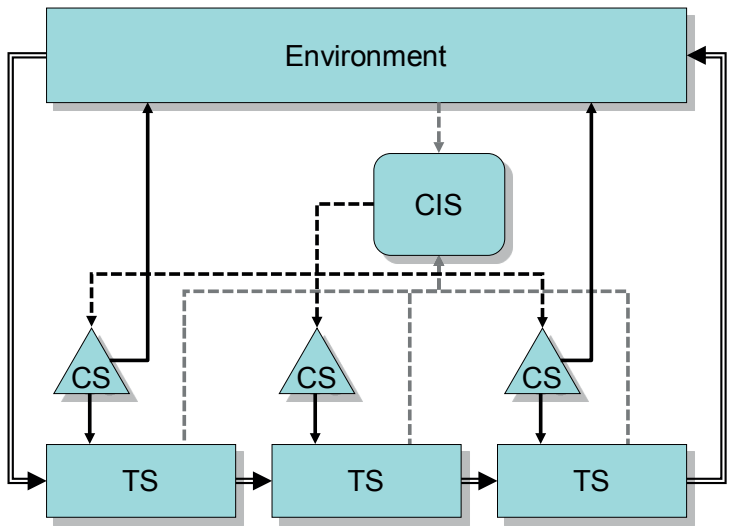

Figure 17. Control model with centralized IS but decentralized CS.

information system (CIS), but with decentralized control systems (CS). This model is shown in Figure 17. We have used two shades of gray to distinguish upward (to CIS) and downward (from CIS) information flows. The control flows from the CSs to the environment correspond with those in Figure 9.

\section{Chains with hybrid control}

Fully decentralized control (choreography) and fully centralized control (orchestration) have serious limitations. Choreography severely limits overall transparency of chains. Orchestration severely limits the autonomy of individual organizations in the chain. Therefore, a hybrid approach often is most suitable (despite its complexity): hybrid control.

\subsection{The hybrid control model}

To obtain a hybrid chain control model, we merge the decentralized and centralized control models that we have introduced in the previous two sections. In doing so, we superimpose centralized control on decentralized control.

We see the result in Figure 18 and Figure 19. The control model in Figure 18 has no explicit peer-topeer information passing (based on the model in Figure 8). The model in Figure 19 includes explicit peer-to-peer information passing (based on the model in Figure 9). Note that in both figures, we have omitted the decision flows from local control systems to the environment for the sake of clarity of the figures.

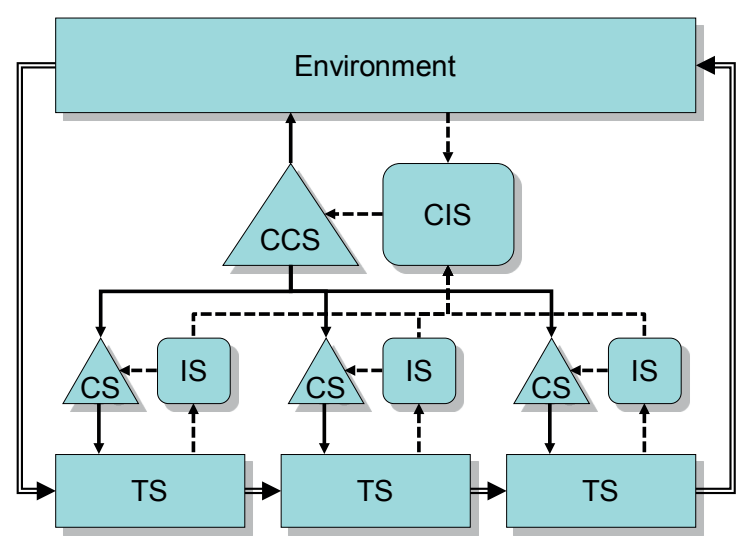

Figure 18. Hybrid control model without $\mathrm{P} 2 \mathrm{P}$ information passing.

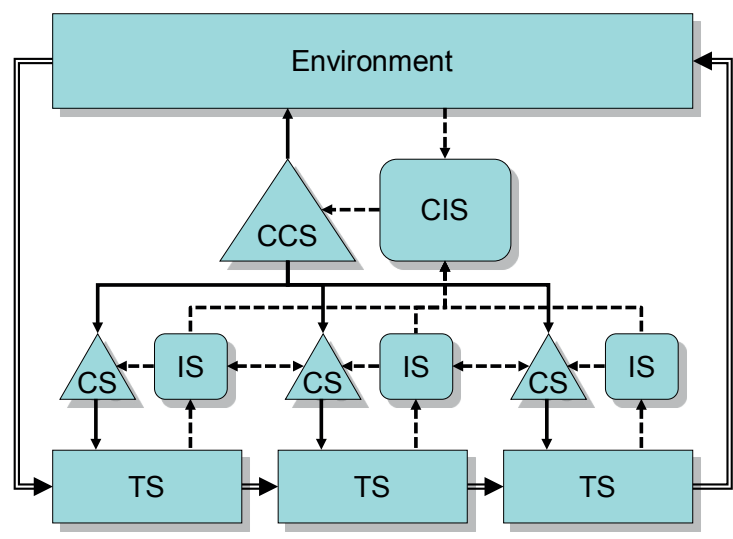

Figure 19. Hybrid control model with $\mathrm{P} 2 \mathrm{P}$ information passing.

\subsection{Feedback loops in the hybrid chain control model}

With the hybrid control model, we distinguish between internal and external feedback loops (as we have done before with the other control models).

For an internal feedback loop, we have in principle the option to handle things in the decentralized (as in Figure 10) or the centralized way (as in Figure 14). To obtain maximal efficiency in chain control, typically the centralized way is best as this involves the smallest number of communication steps. This is illustrated in Figure 20. Even for a chain of three links, there is an advantage for the centralized approach: centralized requires two external and three internal communication steps (as shown in the figure, excluding material flows), whereas decentralized would require two external and five internal steps. The longer the chain is, the greater the difference becomes between centralized and decentralized.

External feedback loops in the hybrid control model always follow the centralized way in the 


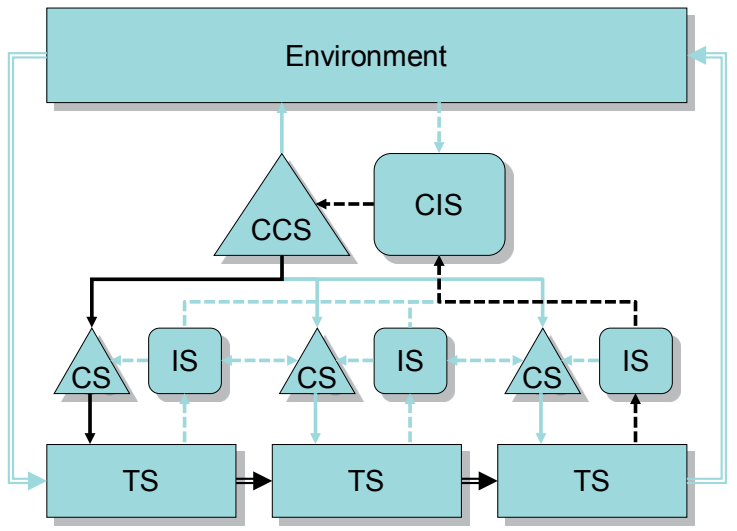

Figure 20. Internal chain-level feedback loop in hybrid control model.

hybrid control model, as the CIS is the only element monitoring the environment for chain control.

\subsection{Choosing the interface level between global and local control}

Using a hybrid chain control model implies that some control decisions are taken at the global (central) level and some at the local (decentral) level. The two levels have to collaborate to make the chain work. This means that choosing the right interface level between global and local control is essential. There are two dimensions of decisions (and the underlying information) to be taken into account here: aggregation and abstraction.

In the aggregation dimension, we determine the granularity of the information passed from IS to CIS and hence the granularity of control decisions of CSS respectively $\mathrm{CS}$. In the granularity dimension, we can further distinguish between the sub-dimensions of time-granularity and goods-granularity (which are usually not completely orthogonal). Time-granularity determines the time scope of information and decisions, e.g., an hour or a day. Goods-granularity determines the granularity of the goods that are the basis for decision making, e.g., an individual goods item, a pallet, or a container. The more detailed the interface between the global and the local level, the more transparency exists in the chain and the more real-time global control becomes possible.

In the abstraction dimension, we determine the concreteness of the information passed from IS to CIS and hence the concreteness of control from CSS to CS. Abstraction only pertains to goods (time-abstraction is not useful). If the abstraction level is high, information only mentions abstract characteristics of goods (such as count, size or weight). If the abstraction level is low, concrete characteristics of goods are mentioned as well (e.g., type numbers of products). The more concrete the interface is between the global and the local level, the more transparency exists in the chain.

Both decision dimensions can be linked to the decision levels as shown in Figure 6: the more abstract and aggregated the decisions (and the underlying information), the higher they are located in the decision pyramid. This is illustrated in Figure 21.

In this division space, we can try and make the divide between decisions that are taken in a centralized fashion in the hybrid control model and those that are taken in a decentralized fashion. Depending on the overall chain control strategy that we take, different divides can be made.

A possible model for this with an emphasis on operational global chain control (as for example discussed by Muckstadt et al. (2001) to obtain tightlycoupled chains) is shown in Figure 22. In this model, we see that the most strategic chain-level choices are always made by individual partners in a chain and the most operational chain-level choices always at the central level. Strategic choices relate to partner autonomy in a chain, hence they are decentralized. Operational choices relate to concrete, real-time chain coordination, hence they are centralized. Obviously, partner-level operational choices are made in a decentralized fashion. The intermediate level choices can be divided in several ways, as shown by the three alternative divides. With divide 1, the central level only handles the most concrete and detailed information, i.e., only controls the real-time, low-level operations. With divide 3, the decentralized level is responsible for taking high-level decisions only and leaves the rest to the centralized level. In this situation, the decentralized level takes the strategic/ tactical decisions and parameterizes the centralized level with these to perform the operational tactical chain control.

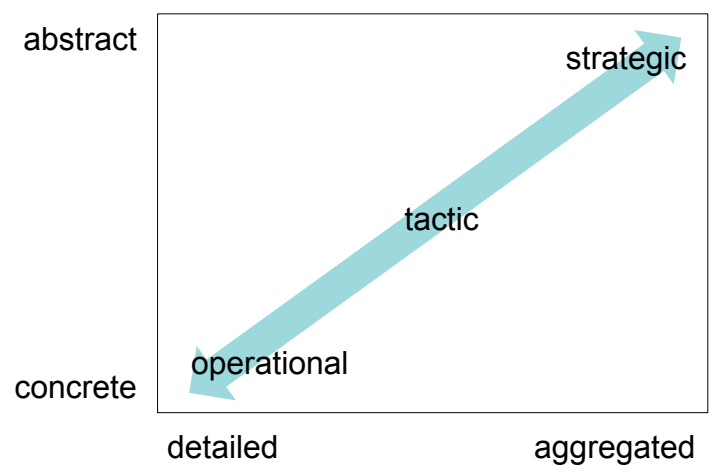

Figure 21. Decision making space. 


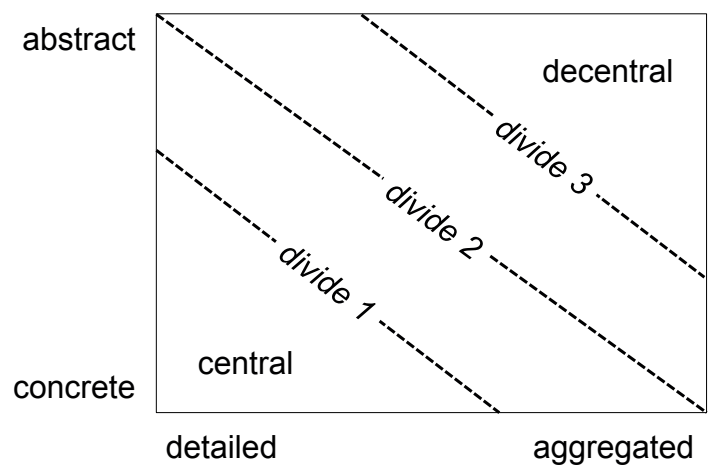

Figure 22. Central/decentral divides in decision space.

\subsection{Evaluation of the hybrid chain control model}

The hybrid chain control model solves the efficiency problems of the decentralized model and the autonomy problems of the centralized model. The penalty for this is added complexity, both in design and execution. As we have illustrated with the discussion in the previous subsection, the hybrid model can be parameterized, creating additional chain design issues compared to the decentralized and centralized models. In the execution of a chain with hybrid control, we require the tight collaboration between the global and the local control levels, which implies run-time complexity.

\section{The agility dimension}

In setting up supply chains and their control, built-in agility becomes increasingly important. This is caused by the fact that markets become increasingly dynamic, both in their requirements to products as in the ways they want their products delivered. Building agility into a supply chain means moving from repeated intensive change processes to a flexible configuration that can easily adapt to changing requirements. We see the latter for example in the concept of virtual factories (Upton and McAfee, 1996; Schulte et al., 2012), in which flexible configurations of production units and the chains that connect them are a starting point.

In realizing chain agility, a number of aspects have to be taken into account. In this section, we briefly address a few important aspects and relate them to the models we have developed in this paper so far. The idea is to briefly sketch a picture, not to be complete here.

\subsection{The business network aspect}

An important aspect to chain agility is to be able to flexibly change the structure of a chain, i.e., to arrive at the concept of dynamic business networks (Grefen et al., 2009; Grefen et al, 2013). In supply chain terms, this means the ability to easily replace a link, add a link or delete a link from a chain.

To dynamically create and change chains, the potential partners for chains are present in a collaborative business ecosystem, in which they can be found based on specific criteria and in which they can be easily coupled to collaborative networks (Camarinha-Matos, Boucher and Afsarmanesh, 2010). In other words, dynamic binding between organizations must be made possible on the business level. Explicit trust management is an essential element in this.

\subsection{The process and service aspect}

In the complexity that hybrid chain control (as discussed in the previous section) augmented with agility brings, explicit business process management becomes of paramount importance.

In chain-level process management, the operation of the entire chain becomes a business process, in which the chain links are process steps or subprocesses, depending on the visibility of details of the processing in the chain links. In this context, the distinction between external and internal process and data models (Grefen, Ludwig and Angelov, 2003) is important. This distinction is closely related to the interface issues between global and local control as discussed in Section 6.3.

In the context of service-orientation, the links in a chain can be seen as distributed business services, each of which provide part of the overall transformation process of the entire chain. Decentralized chain control then becomes service choreography. Centralized chain control becomes service orchestration.

\subsection{The information systems and IT aspect}

Effectively and efficiently dealing with complex, agile supply chains requires the right levels of automation in advanced information systems. The importance of information systems for supply chain management has already been clearly established quite some time ago, for example by Gunasekaran and Ngai (2004). Research has been performed into selecting the right information technology to 
embody these information systems, for example by Sarkis and Talluri (2004).

The importance of information systems to implement dynamic binding mechanisms in chains (as discussed in Section 7.1) has been clearly established. We see this for example in standards like ebXML (OASIS ebXML Joint Committee, 2006) and RosettaNet (Alonso et al., 2004).

Information systems can (or even should) play an explicit role in business process management and business service management as discussed in the previous subsection. A typical example of this can be found in the CrossWork approach, which we discuss in the next section.

\section{Cases}

In this section, we present three cases that show how the hybrid control model that we have developed in Section 6 can be mapped to advanced real-world situations. The cases are intended to be illustrative in this respect - they are by no means intended to be fully representative of their domains.

\subsection{CrossWork: hybrid control in the automotive industry}

In the CrossWork project, research has been performed towards automated support for flexible supply chains in the manufacturing industry, with case studies in the automotive domain (Grefen et al., 2009; Mehandjiev and Grefen, 2010). Flexible supply chains are realized in the form of Instant Virtual Enterprises (IVEs).

A simplified version of the CrossWork architecture is shown in Figure 23. In the architecture, we see

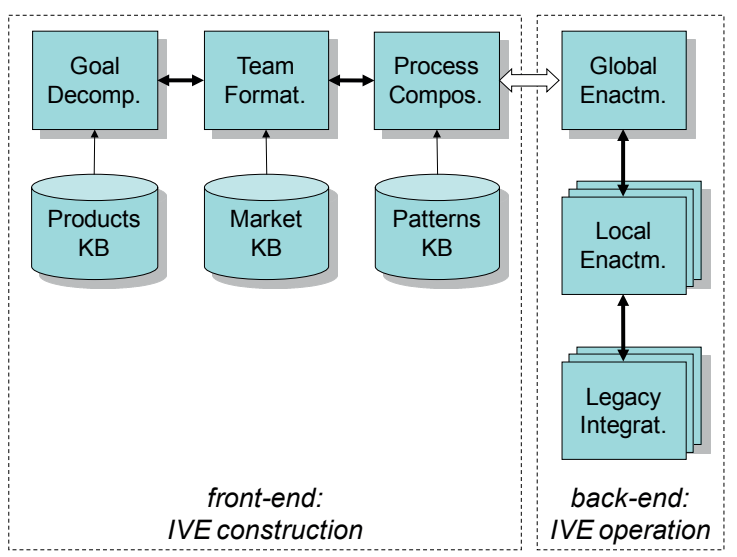

Figure 23. Simplified CrossWork architecture. that the approach explicitly distinguishes between the construction of IVEs (left-hand side of the figure) and the operation of IVEs (right-hand side of the figure).

Tactic chain control with respect to agility is centralized in the design-time part of the supply chain support system: here new chains are formed. Operational business process control at the chain level is centralized - in the architecture this is the responsibility of the Global Enactment module. The Local Enactment modules operate local business processes within the partners of an IVE. In the simplest case of interaction, these local processes are black boxes to the global level. The CrossWork architecture has an interface (Mehandjiev and Grefen, 2010), however, that allows more complex interaction following glass box, half-open box, and open box styles (Grefen et al, 2006), in which global and local level interact in a more fine-grained fashion.

Following the above analysis, the CrossWork approach is conceptually of the hybrid kind: tactical decision making (design time) is centralized, operational decision making (run time) is hybrid (with an emphasis, though, on the central side). Technically, all chain-level synchronization takes place through a centralized orchestration engine. Hence, we classify the Cross Work approach as hybrid control without peer-to-peer information passing. The resulting control model is shown in Figure 24. We see that the CCS module contains both design time and run time support, with corresponding levels in the CIS (the knowledge bases correspond to those in Figure 23). The local CS modules implement the Local Enactment (LE) module functionality (among other things that have been omitted for reasons of clarity). Links between LE modules and environment have been left out for reasons of figure clarity (as in Figure 18).

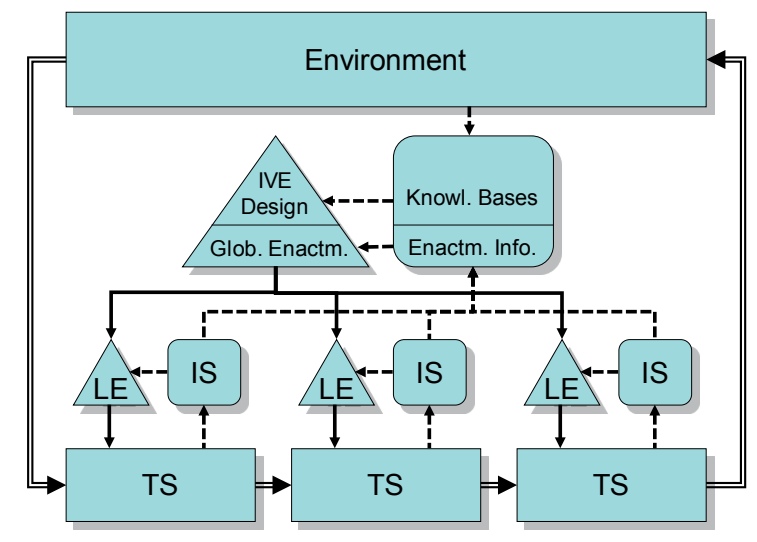

Figure 24. CrossWork hybrid control model. 


\subsection{GET Service: hybrid control in real- time logistics}

In the GET Service project ${ }^{1}$, automated support is developed for real-time management of multi-modal logistics. Real-time information from various parties in the transportation supply chain is used to enable on-the-fly replanning of transport and synchromodality (being able to decide in real-time on the mode of transportation to use between two points). In the GET Service approach, the information system at the operational level is to a large extent centralized in a logistics platform, which functions as an information backbone for all organizations in a chain. The non-real-time perspective of the GET Service chain control model is a standard hybrid model as shown in Figure 19.

In Figure 25, we see the real-time perspective of the GET Service chain control model. Non-realtime elements have been left out for clarity, most notably the local information systems of the links in the chain. Flows between local control systems and the environment have been omitted as well. Local transformation systems (and the environment) feed their real-time process execution data directly into a centralized event warehouse. This event warehouse is used by centralized (chain-level) re-planning and process management decision mechanisms that feed their suggestions to the local control systems of the individual links in the chain. This way, the local information systems of the chain links are bypassed for two reasons: both because they are often not equipped with real-time information processing mechanisms (infrastructure) and because directly feeding data into the central warehouse allows fast integration of data on the chain level (performance).

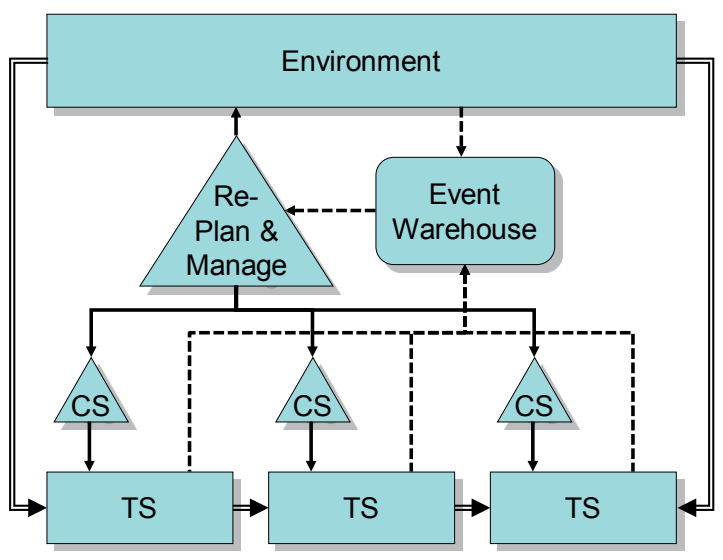

Figure 25. Real-time aspect of GET Service model.

1 http://www.getservice-project.eu
The GET service approach is a hybrid approach, but with different orientation for information management on the one hand and chain control on the other hand. Information management is hybrid, but leans towards centralized, as real-time information management is highly centralized. Chain control is hybrid, but leans towards decentralized, as the CCS mainly suggests the local control systems with respect to important decisions.

\subsection{C: hybrid control across supply chains}

Our third case is also from the logistics domain, but with an emphasis on logistics chain concurrence, i.e., interwoven logistics chains. This interweaving is caused by the fact that companies have to take part in multiple supply chain configurations concurrently (Verdouw et al., 2011). Having interwoven chains, the challenge is to try and optimize them in an overall fashion, e.g., by having chains share resources in a dynamic fashion - such as to not optimize one chain and de-optimize another one in the same go.

To do so, the concept of Cross-Chain Control Center (4C) has been introduced to integrate intra-chain and inter-chain control to achieve collaboration advantages (Dinalog 2013). Intra-chain control is hybrid at the chain level, but inter-chain control even hybrid above the chain level. Hence, a three level control model would be possible, but we prefer to stick to two levels where the $4 \mathrm{C}$ also has the role of the CCS at the level of an individual chain.

This is illustrated in Figure 26. In the figure, we have abstracted supply chain links into black boxes and merged information and control links for reasons of clarity. In the figure, we see two chains SC1 and SC2, which each consist of three links (labeled L1, L2 and L3). One link is shared between the chains, so here

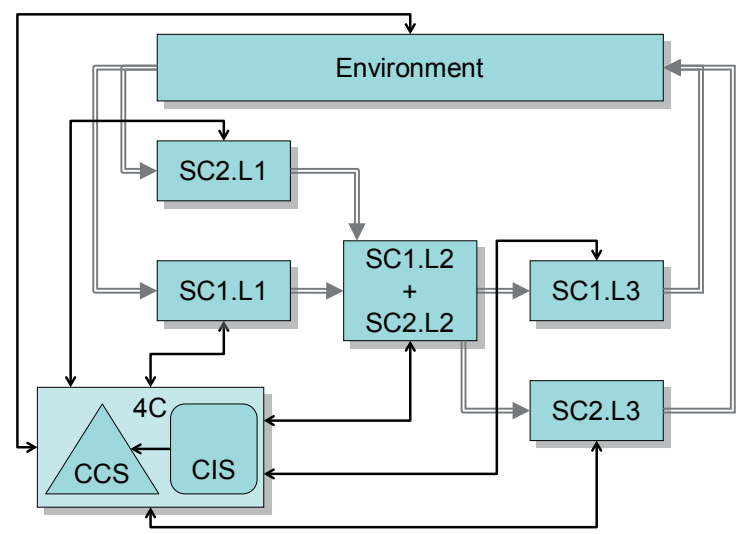

Figure 26. 4C chain integration model. 
the two chains concur. The cross-chain control center (C4) consists of the CIS and CCS, which controls the two individual chains plus the concurrence of the two chains. Cross-chain control can optimize the use of the resources of the shared chain link, and hence of both individual chains.

\section{Conclusions}

In this paper, we have explored the issue of supply chain control, where we have approached control from an information management perspective. We have combined a basic supply chain model and a cybernetic control model in two alternative ways: embedding the latter in the former and the other way around. This has resulted in two extreme chain control models (centralized and decentralized). We have 'interpolated' between these two models to arrive at a hybrid chain control model. The hybrid control model allows parameterization of control, but at the cost of additional complexity. We see this complexity both at design time and at run time of a chain.

Analysis of a number of cases shows that the hybrid control model is often used in advanced contexts as they are often found in $R \& D$ projects. In these projects, however, the parameterization of the hybrid model is typically more or less rigid, as it is statically defined in chain control approach of a project. This indicates that a truly flexible application of the hybrid model in practical, industrial scenarios still requires quite some development.

Flexible application of the model presented in this paper does provide good opportunities to have chain control in complex, dynamic markets adapted well to the requirements of those markets.

\section{References}

Aalst, W. van der (2009a). Choreography. In: Liu, L., \& Özsu, M. (2009). Encyclopedia of Database Systems (pp. 329). Springer. doi:10.1007/978-0-387-39940-9_1198

Aalst, W. van der (2009b). Orchestration. In: Liu, L., \& Özsu, M. (2009). Encyclopedia of Database Systems (pp.2004-2005). Springer. doi:10.1007/978-0-387-39940-9_1197

Alonso, G., Casati, F., Kuno, H., Machiraju, V. (2004). Web Services: Concepts, Architectures and Applications. Springer.

Beer, S. (1984). The Viable System Model: Its Provenance, Development, Methodology and Pathology. Journal of the Operational Research Society, 35:7-25.

Camarinha-Matos, L., Boucher, X., Afsarmanesh, H. (2010). Collaborative Networks for a Sustainable World; In Proceedings 11th IFIP WG 5.5 Working Conference on Virtual Enterprises. St. Etienne, France. doi:10.1007/978-3-642-15961-9

Corswant, F. von, \& Fredriksson, P. (2002). Sourcing trends in the car industry: a survey of car manufacturers' and suppliers' strategies and relations. International Journal of Operations \& Production Management 22(7), 741-758. doi:10.1108/01443570210433526

Dinalog (2013). 4C: Cross Chain Control Center. Dutch Institute for Advanced Logistics. Available at: http://www.dinalog.nl/en/themes/4c_ cross_chain_control_center

Grefen, P., Ludwig, H., \& Angelov, S. (2003). A Three-Level Framework for Process and Data Management of Complex E-Services. International Journal of Cooperative Information Systems, 12(4), 487-531. doi:10.1142/S0218843003000838

Grefen, P., Ludwig, H., Dan, A., Angelov, S. (2006). An Analysis of Web Services Support for Dynamic Business Process Outsourcing. Information and Software Technology, 48(11):1115-1134. doi:10.1016/j.infsof.2006.03.010

Grefen, P., Mehandjiev, N., Kouvas, G., Weichhart, G., Eshuis, R. (2009). Dynamic business network process management in instant virtual enterprises. Computers in Industry, 60(2):86-103. doi:10.1016/j.compind.2008.06.006

Grefen, P. (2010). Mastering E-Business. London: Routledge.

Grefen, P., Lüftenegger, E., van der Linden, E., Weisleder, C. (2013). BASE/X: Business Agility through Cross-Organizational Service Engineering. Beta Working Paper 414. Eindhoven University of Technology.

Gunasekaran, A., Ngai, E. (2004). Information Systems in Supply Chain Integration and Management. European Journal of Operational Research, 159(2), 269-295. doi:10.1016/j.ejor.2003.08.016

Heaney, B. (2012). Supply Chain Visibility Excellence: Mastering Complexity and Landed Costs. Boston: Aberdeen Group.

Hoen, K., Tan, T., Fransoo, J., van Houtum, J. (2012). Effect of Carbon Emission Regulations on Transport Mode Selection under Stochastic Demand. Flexible Services and Manufacturing Journal (Published Online). doi:10.1007/s10696-012-9151-6

Jansen, B., Swinkels, P., Teeuwen, G., van Antwerpen de Fluiter, B., Fleuren, H. (2004). Operational planning of a large-scale multi-modal transportation system. European Journal of Operational Research, 156(1), 41-53. doi:10.1016/j.ejor.2003.02.001

Luftenegger, E., Grefen, P., Weisleder, C. (2012). The Service Dominant Strategy Canvas: Towards Networked Business Models. In Proceedings $13^{\text {th }}$ IFIP Working Conference on Virtual Enterprises (pp. 207-215). Bournemouth, UK. doi:10.1007/978-3-642-32775-9_21 
Grefen, P., \& Dijkman R.

Lusch, R., Vargo, S., O'Brien, M. (2007). Competing through service: insights from service-dominant logic. Journal of Retailing, 83(1), 5-18. doi:10.1016/j.jretai.2006.10.002

Maxton, G.P., Wormald, J. (2004). Time for a Model Change: Re-engineering the Global Automotive Industry. New York: Cambridge University Press. doi:10.1017/CB09780511488535

Mehandjiev, N., Grefen, P. (2010). Dynamic Business Process Formation for Instant Virtual Enterprises. Springer London. doi:10.1007/9781-84882-691-5

Muckstadt, J., Murray, D., Rappold, J., Collins, D. (2001). Guidelines for Collaborative Supply Chain System Design and Operation. Information Systems Frontiers, 3(4), 427-453. doi:10.1023/A:1012824820895

OASIS ebXML Joint Committee (2006). The Framework for eBusiness. OASIS.

Olhager, J. (2012). The Role of Decoupling Points in Value Chain Management. In: Jodlbauer, H., Olhager, J., Schonberger, R. (Eds.). Modelling Value. Springer. doi:10.1007/978-3-7908-2747-7_2

Porter, M. (1985). Competitive Advantage: Creating and Sustaining Superior Performance. New York: Free Press.

Sarkis, J., Talluri, S. (2004). Evaluating and Selecting e-Commerce Software and Communication Systems for a Supply Chain. European Journal of Operational Research, 159(2), 318-329. doi:10.1016/j.ejor.2003.08.018

Schulte, S., Schuller, D., Steinmetz, R., Abels, S. (2012). Plug-and-Play Virtual Factories. IEEE Internet Computing, 16(5), 78-82. doi:10.1109/ MIC.2012.114

Smith, S., Jiao, R., Chu., C. (2012). Editorial: advances in mass customization. Journal of Intelligent Manufacturing (Published Online). doi:10.1007/s10845-012-0700-3

Upton, D., McAfee, A. (1996). The Real Virtual Factory. Harvard Business Review, 74(4), 123-133.

Verdouw, C., Beulens, A., Trienekens, J., van der Vorst, J. (2011). A framework for modelling business processes in demand-driven supply chains. Production Planning \& Control, 22(4), 365-388. doi:10.1080/09537287.2010.486384 